\title{
Concept for Unmanned Microfluidic Reactor for the Optimization and Production of Well-defined Nanoparticles
}

\author{
Jacinto Sá \\ Department of Chemistry - Ångström Laboratory, Uppsala University, Sweden \\ Institute of Physical Chemistry, Polish Academy of Sciences, Warsaw, Poland \\ *Corresponding Author: jacinto.sa@kemi.uu.se
}

Copyright $(2014$ Horizon Research Publishing All rights reserved.

\begin{abstract}
Nanoparticles are used, or being evaluated for use, in many fields, such as medicine, manufacturing, materials, energy, electronics, and environment. The characteristics of nanocrystals are strongly influenced by their physical dimensions and shape, which are tune by adjusting a plethora of experimental conditions, such as reagents concentration, reaction temperature and time. This demands for a large number of experiments to be carried out, mainly using a trial and error methodology. This publication describes a conceptual design of an unmanned microfluidic reactor for the optimization and production of nanoparticles with predefined physicochemical properties, with special emphasis on the production of gold group metal particles.
\end{abstract}

Keywords Nanoparticles, Microfluidics, Automated, Optimization

\section{Introduction}

Nanoparticles (NPs) have one dimension that measures 100 nanometers or less. Their properties often differ from their larger counterpart materials because of their greater surface area and surface reactivity. Thus, NPs are used, or being evaluated for use, in many fields, such as medicine, manufacturing, materials, energy, electronics, and environment. The characteristics of nanocrystals are strongly influenced by their physical dimensions and shape [1]. Thus, in order to answer fundamental questions and develop future applications, one needs to prepare particles with well-define size and shape.

There are two main routes for NPs formation: top-down and bottom-up approaches. In top-down routes, nanometer-sized structures are engineered from bulk materials using a combination of lithography, micromachining methods, and etching [2]. Such approaches are technically challenging and although reproducible they do not readily lend themselves to large-scale production. A bottom-up approach involves the chemical growth of particles on an atom-by-atom basis until the desired particle size and shape are achieved. The growth process occurs spontaneously in super-saturated solutions and has been successfully used to create high-quality spherical, cubic, tubular, and tetrahedral crystallites in kilogram quantities and above [3]. Another factor for the popularity of the bottom-up approach is that it can be carried out with standard synthetic chemistry lab techniques, which are versatile and ease to use. A variety of sophisticated chemical strategies have been reported for producing near defect-free nanoparticles of consistent size, shape, and chemical composition. Their implementation, however, remains a complex and difficult undertaking that requires a combination of skill, intuition, and extensive experimentation to obtain well-defined nanoparticles with tightly specified properties [4]. It remains a formidable challenge to prepare high-quality NPs in a controlled and reproducible manner, which is recognized to be the foremost obstacle to the full exploitation of many nanoscale phenomena.

In simple terms, NPs are formed via a two-stage process, namely nucleation phase followed gradual growth phase. Unfortunately, in most cases, nucleation and growth occur concurrently, and thus the final particle population will therefore exhibit a broad (and undesirable) size distribution. Accordingly, to obtain monodisperse particles, it is necessary to set up conditions in which all nucleation takes place over a short period of time with additional material being supplied so gradually that it find its way to the nuclei without the solute concentration reaching a level at which further nucleation can take place. In practical terms it is crucial that all nuclei should form and grow in an identical environment with state functions (such as temperature, pressure, and concentration) assuming constant values throughout the reaction volume. In conventional syntheses within bulk reactors (where turbulent mixing is used to ensure rapid combination of reagents) significant variations in physical conditions across the reaction chamber are 
normal, thus generating wide particle-size distributions.

Microfluidic systems, which allow for rapid and controlled thermal and mass transfer, are ideal for nanoparticle production. In simple terms, microfluidic devices manipulate and process sub-microliter volumes of liquid in enclosed channels that are typically no more than a few hundred microns in diameter. Importantly for chemical synthesis, they have a number of advantageous features, including precise control over reaction conditions, rapid heating, cooling and mixing of fluid streams, and the ability to combine multiple chemical processes into a single, integrated device [5].

This publication proposes a concept for the development of an automated unmanned microfluidic setup able to optimize and produce NPs with desired physicochemical properties on-demand, with special focus on gold group metals NPs. The proposed 'black-box' automated reactor aims to circumvent the current problems in obtaining monodispersed in size and homogeneous in shape NPs. Moreover, the optimized synthesis procedures can be standardized and used by others, for on-demand synthesis of tailored NPs.

\section{Concept}

The proposed setup is schematically represented in figure 1. Briefly, the process starts with the specification of NPs requirements, in respect to size, shape and in the case of gold group metal NPs optical properties. The setup evaluates a multiple reaction conditions until it eventually identifies an appropriate set of reaction conditions that yields particles with the specified properties, and it would then continue to produce particles until instructed to stop. There are three essential parts to any automated system, namely (i) physical machinery to perform the process at hand, (ii) online detectors for monitoring the output of the process, and (iii) decision-making software that repeatedly updates the process parameters until a product with the desired properties is obtained.

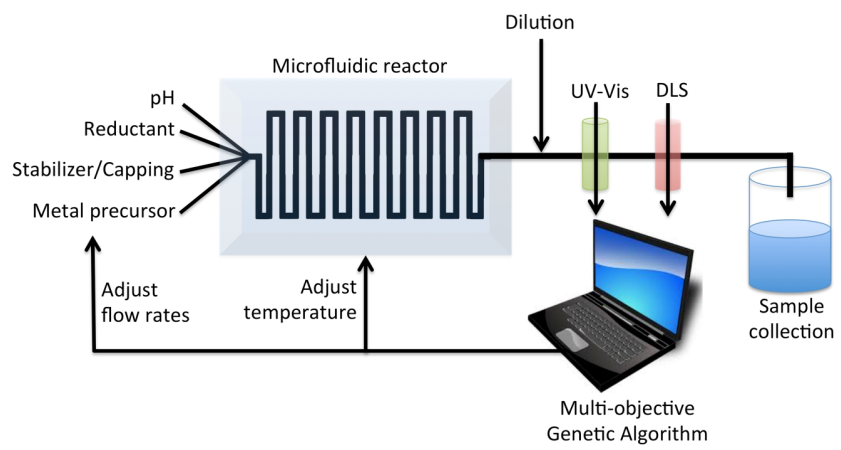

Figure 1. Schematic representation of automated microfluidic reactor for on-demand NPs production with desired physicochemical properties.

The key aspects of each part are succinctly described below.

\section{i) Physical machinery}

The machinery should provide precise reproducible control of the physical process and should carry out the individual process steps as rapidly as possible to enable fast screening. A Y-shaped microfluidic reactors comprised of four inlet channels, into which reagents are injected using precision syringe pumps and capillary connectors, should provide sufficient variability and flexibility to optimize the process. Varying the volumetric flow rates of the reagent solutions will control the composition of the resultant mixture and residence time inside the microfluidic chip. The microfluidic chip is connected to a climate control unit able to heat uniformly the reaction mixture to temperature up to $250{ }^{\circ} \mathrm{C}$. The starting solutions can be preheated before injection, either by heating their vessel or by passing the solution through a heated coil [6]. During the optimization procedure, the resultant NPs solution needs to be diluted at the outlet in order to be at the optimal concentration range for the online detectors analysis. At any time with this configuration, five variables can be changed and optimized, namely flow of each reagent and reaction temperature, conferring the setup high versatility and applicability.

\section{ii) Online detectors}

The online detectors should provide real-time low-noise information about the end product. Two detection tools, namely UV-Vis absorption and Dynamic Light Scattering (DLS), assembled in series after the reactor outlet are used to provide continuous and real-time analysis. This combination expands significantly the number optimization parameters. The UV-Vis absorption analysis provides information of absorption peak wavelength $\left(\lambda_{\max }\right)$, intensity and full-width half-maximum. DLS analysis yields information about NPs size, sample homogeneity and in specific cases NPs shape. It should be mentioned that nanoparticle formation is naturally slow [7], thus it does not require extremely fast online detection. Finally, the setup can easily accommodate other online detectors either as add-ons or as replacement to the existent ones.

\section{iii) Decision-making software}

The decision-making software should search for the optimal conditions in a way that is both parsimonious in terms of experimental measurements (in order to ensure a fast time-to-solution) and tolerant of noise in the experimental system. Experimental noise relates to slightly different products due to mechanical imperfections in the reactor and temporal chemical decay in the starting reagents. Commonly, NPs synthesis automation uses the so-called utility function that reduces all of the known information about the particles to a single figure of merit that characterizes the particle quality, usually defined in such a way that the figure of merit decreases steadily to zero as the measured properties get progressively closer to the desired properties. The prime reason to decrease the optimization to a single figure of merit is to decrease computational time, 
and to take advantage of recently developed minimization routines, such as the simplex method [8], which can perform constrained optimization of expensive noisy black-box functions. Black-box is a device, system or object which can be viewed in terms of its input, output and transfer characteristics without any knowledge of its internal workings. The opposite of a black-box is a system where the inner components or logic are available for inspection. Black-box methods will always converge to an optimum but there is no guarantee it will find the global optimum, which may be located in a distant unexplored region of the parameter space. To stand a better chance of finding the global optimum, different optimization routines are required.

A possible strategy to reach global optimum is to perform a multi-objective optimization. Multi-objective genetic algorithm (MO-GA) is capable of solving multi-objective optimization problems by finding an evenly distributed set of points on the Pareto front. This approach can be used to optimize non-smooth and noisy problems (current case), and permits the use of bound and linear constraints on input variables and tolerance values. Briefly, the MO-GA works on a population using a set of operators that are applied to the population. A population is a set of points in the design space. The initial population is generated randomly by default. The next generation of the population is computed using the non-dominated rank and a distance measure of the individuals in the current generation. The MO-GA uses a controlled elitist genetic algorithm that favors individuals with better fitness value (rank) as well as individuals that can help increase the diversity of the population even if they have a lower fitness value [9]. It is important to maintain population diversity for convergence to an optimal Pareto front. The MO-GA optimization can be carried out in commercial available software and toolboxes, such as Matlab's Global Optimization Toolbox. Apart of taking advantage of existent algorithms, Matlab's Global Optimization toolbox also enables the use of parallel computing and hybrid schemes that can speed-up the finding of the optimal Pareto front. Recently, this methodology was used to find and optimize $\mathrm{Au}_{2} \mathrm{O} \mathrm{X}$-ray absorption spectrum, an unstable structure formed during $\mathrm{Au}_{2} \mathrm{O}_{3}$ reduction [10].

An optimization routine, involves predefining starting materials concentration, constraining input variables (upper and lower limits for reagents flow and reactor temperature), establishing desired target values (global optimum) and tolerance values. The system will optimize the synthesis procedure by changing the reaction conditions (reagent flows and temperature), evaluating the results of each interaction (output values of online detectors) and comparing them to the target values. The process will continue until it finds an optimal Pareto front, providing the experimental conditions for producing NPs with desired physicochemical properties. The number of interactions depends on tightness of the tolerance values and/or complexity of the desired NPs.

\section{Summary}

This publication describes the conceptual design of an unmanned microfluidic reactor for the production of well-defined NPs. It has the ability to optimize and produce particles on demand, and deliver standardized synthesis procedures for production of similar NPs in other laboratories. To finalize, it is important to mention that all the required components for the development of this setup are commercially available.

\section{REFERENCES}

[1] C. D. Donega, S. G. Hickey, S. F. Wuister, D. Vanmaekelbergh, A. Meijerink, J. Phys. Chem. B 107 (2003) 489 .

[2] D. Y. Xia, D. Li, Z. Ku, Y. Luo, S. R. J. Brueck, Langmuir 23 (2007) 5377.

[3] a) O. Masala, R.Seshadri, Annu. Rev. Mater. Res. 34 (2004) 41; b) J. Park, K. An, Y. Hwang, J.-G. Park, H.-J. Noh, J.-Y. Kim, J.-H. Park, N.-M. Hwang, T. Hyeon, Nat. Mater. 3 (2004) 891.

[4] a) C. D. Dushkin, S. Saita, K. Yoshie, Y. Yamaguchi, Adv. Colloid Interface Sci. 88 (2000) 37; b) C. N. R. Rao, V. V. Agrawal, K. Biswas, U. K. Gautam, M. Ghosh, A. Govindaraj, G. U. Kulkarni, K. P. Kalyanikutty, K. Sardar, S. R. C. Vivekchand, Pure Appl. Chem. 78 (2006) 1619; c) G. G. Yordanov, G. D. Gicheva, B. H. Bochev, C. D. Dushkin, E. Adachi, Coll. Surf. A-Physicochem. Eng. Asp. 273 (2006) 10.

[5] A. J. deMello, Nature 442 (2006) 394.

[6] H. Z. Wang, X. Y. Li, M. Uehara, Y. Yamaguchi, H. Nakamura, M. P. Miyazaki, H. Shimizu, H. Maeda, Chem. Commun. (2004) 48.

[7] S. Krishnadam, A. Yashina, A. J. deMello, J. C. deMello, Adv. Chem. Eng. 38 (2010) 195.

[8] T. G. Kolda, R. M. Lewis, V. Torczon, SIAM Rev. 45 (2003) 385.

[9] K. Deb, Multi-Objective Optimization using Evolutionary Algorithms, John Wiley \& Sons ISBN 047187339.

[10] J. Szlachetko, J. Sá, M. Nachtegaal, U. Hartfelder, J.-Cl. Dousse, J. Hoszowska, D. L. A. Fernandes, H. Shi, C. Stampfl, J. Phys. Chem. Lett. 5 (2014) 80. 